\title{
COVID-19 aus Sicht der Praxen und Notaufnahmen
}

Tobias Herold, Markus Wörnle, Jörg Schelling

\author{
COVID-19 stellt die Praxen und Notaufnahmen in ganz Deutschland vor große Schwierig- \\ keiten. Außer der Beachtung von Hygiene und Isolation ist vor allem die korrekte Zuwei- \\ sung von Patienten zur ambulanten, stationären oder intensivmedizinischen Therapie \\ eine Herausforderung. Dieser Beitrag bietet eine Übersicht zu Aspekten der Betreuung \\ und der Risikoeinschätzung des Erkrankungsverlaufs bei COVID-19-Patienten.
}

\section{Hintergrund}

Die durch SARS-CoV-2 verursachte Coronavirus-Erkrankung (COVID-19) wird hauptsächlich durch Tröpfchen übertragen [1], und im Schnitt steckt ein Infizierter mehr als 3 weitere Menschen an. Zur Infektionsvermeidung und zum Schutz der Mitarbeiter im Gesundheitssystem sind besondere Hygienemaßnahmen und Schutzkleidung erforderlich.

COVID-19 zeigt einen sehr heterogenen Verlauf. Mehrheitlich werden nahezu asymptomatische Patienten beobachtet, aber auch solche, die in kürzester Zeit ein Lungenversagen entwickeln. Neben Symptomen einer Atemwegsinfektion können zum Beispiel auch Elektrolytentgleisungen bei Durchfällen oder thromboembolische Ereignisse auftreten.

\footnotetext{
Merke

Eine Einteilung in ambulant behandelbare Patienten und solche, die einer stationären Behandlung bedürfen, ist ohne etablierte Algorithmen schwierig [1].
}

Die hohe Infektiosität und der heterogene und teilweise schwere Krankheitsverlauf machen die Versorgung von COVID-19-Patienten zur Herausforderung in Praxen und Notaufnahmen. Teilbereiche sollen in dem folgenden Artikel hervorgehoben werden.

\section{Hausarztpraxen}

\section{Zu Beginn der Pandemie}

In den Hausarztpraxen stand zu Beginn der Pandemie praktisch nirgends Schutzkleidung zur Verfügung, denn bisher kam es in der Primärversorgung nicht regelhaft zur Betreuung von potenziell hochinfektiösen Patienten. In der Influenzasaison wurde auf ausreichende Händehygiene und eine Impfung aller Praxismitarbeiter geachtet. Einzelne Praxen verfügten noch über (abgelaufene) einzelne Schutzkittel aus der Zeit der H1/N1-Pandemie. Nach und nach mussten die Praxen sich also Schutzmas- ken, Handschuhe und Desinfektionsmittel beschaffen. In einzelnen Landkreisen konnten hier auch der Katastrophenschutz bzw. das Landratsamt aushelfen.

Von den Kassenärztlichen Vereinigungen war zu Beginn wenig Hilfe zu erhalten [2]. Schutzmittel wurden in der Reihenfolge Krankenhaus - Pflegeheim - Arztpraxis priorisiert. Auch in Notaufnahmen und Krankenhäusern zeigte sich bereits zu Beginn der Pandemie, dass enorme Anpassungen zur Versorgung der Vielzahl von hochinfektiösen Patienten notwendig waren. Krisenstäbe zur Veränderung der Abläufe und zur Beschaffung von Schutzkleidung wurden nahezu überall gegründet.

\section{Merke}

Um für weitere Infektionswellen besser gerüstet zu sein, erscheint hier eine Anpassung der bestehenden Pandemiepläne sowie eine entsprechende Bevorratung von Schutzkleidung für Notfälle wichtig.

\section{Strukturelle Veränderungen und Schutzmaß- nahmen}

In vielen Praxen wurden aus privaten Mitteln Plexiglasscheiben oder -wände installiert. Hier waren regelmäßige Rundmails und Informationen in den MMK-Benefits der DEGAM (Deutsche Gesellschaft für Allgemein- und Familienmedizin) hilfreich [3]. Inzwischen verfügen die meisten Praxen über mechanischen Schutz, Masken für Mitarbeiter, Handschuhe und Desinfektionsmittel. Dort, wo es räumlich möglich war, wurden einzelne Wartebereiche und Sprechzimmer ausschließlich für Abstriche oder eine Infektionssprechstunde eingerichtet.

Patienten wurden gebeten, sich ausschließlich nach telefonischer Voranmeldung in die Praxen zu begeben. Entsprechende Merkblätter wurden ausgelegt oder im Internet veröffentlicht [4]. Falls eine Trennung zwischen normalem Praxisbetrieb und einer „Corona-Sprechstunde“ nicht möglich war bzw. ist, besteht inzwischen die Möglichkeit, an spezielle Corona-Bereitschaftspraxen zu verweisen, die auf Landkreisebene eingerichtet wurden und an denen sich im Regelfall mehrere Hausarztpraxen 
beteiligen [4, 5]. Im direkten Patientenkontakt müssen alle Mitarbeiter Masken und Handschuhe tragen.

\section{Notaufnahme}

\section{Strukturelle Veränderungen}

Für das Management infektiöser Patienten in den Notaufnahmen ist vor allem eine frühzeitige Erkennung und Trennung von potenziell infektiösen Patienten von den übrigen Patienten notwendig, um eine weitere Erregerübertragung zu vermeiden. Hier haben sich Sicherheitskontrollen und vorgeschaltete Check-Points bewährt. An diesen werden potenziell infektiöse Patienten mittels fokussierter Fragen nach Symptomatik und Temperaturmessung erfasst und die Patientenströme entsprechend geleitet.

\section{Merke}

Jeder Patient sollte unmittelbar beim Betreten der Klinik mit einem chirurgischen Mund-Nasen-Schutz (MNS) ausgestattet werden.

Eine generelle Maskenpflicht bei gleichzeitigem Verbot privater Masken vermeidet hier potenzielle Risiken, die durch eine inzwischen unüberschaubare Vielzahl an Masken entsteht. In den Notaufnahmen erfolgt dann die Triage mit den bestehenden Ersteinschätzungsverfahren nach Behandlungsdringlichkeit.

\section{Schutzmaßnahmen}

Prinzipiell sollte nur Personal eingesetzt werden, das im Umgang mit Hygienemaßnahmen geschult ist. Das Personal muss eine persönliche Schutzausrüstung (PSA) tragen, bestehend aus Schutzkittel, Einweghandschuhen, dicht anliegendem MNS/ggf. Atemmaske und Schutzbrille.

\section{Merke}

Bei der direkten Versorgung von Patienten mit wahrscheinlicher oder bestätigter COVID-19-Infektion müssen FFP2-Masken getragen werden.

Die genannten Maßnahmen führen zu einem großen zeitlichen Mehraufwand in der Erstversorgung der Patienten und einem immensen Verbrauch von Schutzmaterial:

- Die Patienten müssen in der Notaufnahme in einer Einzelbox unter Isolationsbedingungen versorgt werden.

- Transporte innerhalb der Klinik sollten auf das Notwendigste reduziert werden und, falls unabdingbar, als Einzeltransport mit MNS für den Patienten erfolgen.

- Das Personal trägt PSA mit FFP2-Masken.

- Erfolgt eine stationäre Aufnahme, so muss eine Einzelunterbringung in einem Isolierzimmer erfolgen.
- Eine gemeinsame Isolierung mehrerer positiv getesteter Patienten ist unter bestimmten Bedingungen möglich.

- Verdachtspatienten dürfen jedoch nicht zusammen untergebracht werden

- In jedem Fall ist zu beachten, dass nach Entlassung oder Verlegung der Patienten vor dem Wiederbenutzen der Räumlichkeiten eine Schlussdesinfektion erfolgen muss (siehe RKI-Empfehlungen).

\section{Organisatorische Probleme}

Die organisatorischen Abläufe werden durch methodische Probleme in der Diagnosesicherung erschwert. Der Nachweis der Erkrankung erfolgt über eine PolymeraseKettenreaktion (PCR). Hierbei ist zu berücksichtigen, dass in einigen Fällen ein negativer Befund COVID-19 noch nicht definitiv ausschließt.

\section{Cave}

Solange kein definitiver Infektionsausschluss erfolgt ist, müssen Verdachtspatienten ebenso behandelt werden wie Patienten mit Nachweis der Infektion.

Mittlerweile stehen flächendeckend ausreichend Testkapazitäten zur Verfügung. Allerdings gibt es methodische Limitationen im Testverfahren, die zwangsläufig eine Verzögerung bis zum Erhalt des Testergebnisses mit sich bringen. In den meisten Notaufnahmen liegen die Ergebnisse der Testung verfahrensbedingt zwar erst nach mehreren Stunden, aber noch am selben Tag vor. Dies ist aber nicht für alle Notaufnahmen und Praxen der Fall.

\section{Verzahnung ambulanter und stationärer Bereich}

Besonders problematisch erscheint in der aktuellen Krise die mangelnde Verzahnung von ambulantem und stationärem Bereich im Rahmen von Infektionskrankheiten (Infobox 1). Hier können lokale Netzwerke und persönliche Kontakte teilweise Abhilfe schaffen - denn eine strukturelle und organisierte Betreuung einer großen Anzahl von infektiösen Patienten gab es vor der Corona-Pandemie nicht.

\section{INFOBOX 1}

Es erscheint zwingend notwendig, diese Strukturen und Abläufe zu analysieren, kritisch zu hinterfragen und im Verlauf der nächsten Monate zu ändern, um in Zukunft eine reibungslose Versorgung betroffener Patienten zu gewährleisten.

Dies erfordert

- eine Überarbeitung der entsprechenden Pandemiepläne,

- die Bevorratung entsprechender Schutzausrüstung, 
- standardisierte Übergabe- und Einweisungsprotokolle und -wege,

- regelmäßige runde Tische, gemeinsame Fortbildungen und Übungen sowie

- ein funktionierendes Entlass-Management.

\section{Die SARS-CoV-2-Infektion}

\section{Symptome}

Nach Infektion mit SARS-CoV-2 treten nach meist 4-5 Tagen erste Krankheitszeichen auf. Eine Inkubationszeit von > 12 Tagen ist sehr ungewöhnlich [6]. Während sich in der ambulanten Versorgung die Anamnese zu Beginn auf die „klassischen“ Symptome wie Husten und Fieber konzentrierte, zeigte sich bald, dass viele Patienten entweder keine Symptome hatten oder allgemeine Schwäche oder Geruchs- und Geschmacksverlust beklagten. Die Reiseanamnese wurde zunehmend irrelevant.

\section{Merke}

Typische Erkrankungszeichen einer SARS-CoV-2-Infektion existieren nicht.

Es werden vor allem Fieber, Husten und Halsschmerzen sowie Geruchs- und Geschmacksstörungen beobachtet. Allerdings ist auch eine Vorstellung mit Brust- oder Kopfschmerzen oder einer gastrointestinalen Symptomatik mit z. B. Durchfällen möglich (Infobox 2) [7-9]. Daher ist für eine Einschätzung der Wahrscheinlichkeit einer Infektion mit SARS-CoV-2 immer die Kenntnis der regionalen Häufigkeit von COVID-19-Fällen wichtig.

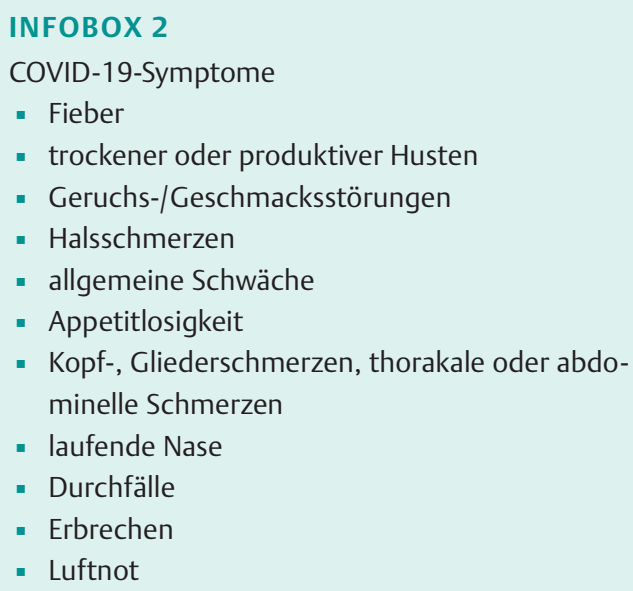

\section{Diagnostik von COVID-19}

Die Diagnose wird über einen Nachweis des Virus mittels PCR gestellt. Hierfür wird in der Regel respiratorisches Material untersucht, dabei sind falsch negative Ergebnis- se aber nicht selten. Gerade bei Zeichen einer Infektion des unteren Atemtraktes scheinen Proben aus den tieferen Atemwegen (Sputum) eine höhere Genauigkeit zu besitzen [10]. Serologische Antikörpertests werden bislang nur selten angewendet und sind bisher keine Leistung der gesetzlichen Krankenkassen. Laborabnahmen werden zunehmend ambulant durchgeführt, hier hat sich aber bisher kein einheitlicher Standard etabliert.

Merke

Wenn bei bestehendem Verdacht auf COVID-19 ein negativer PCR-Test vorliegt, sollte zunächst die Hygiene/ Isolation fortgeführt werden und ein erneuter Test erfolgen.

In der Notaufnahme erfolgt meist eine breite Labordiagnostik:

- Häufig beobachtete Laborveränderungen sind eine Lympho- und Eosinopenie.

- Erhöhte Entzündungszeichen (CRP, Ferritin und IL-6) und eine erhöhte LDH finden sich bei schweren Verläufen.

- Procalcitonin (PCT) ist in der Regel nicht erhöht.

- Bei kritisch kranken Patienten wird häufig eine Erhöhung von Troponin und D-Dimer beobachtet [11]

In der Bildgebung scheint die Thorax-Sonografie eine hoffnungsvolle Methode zu sein. Einzelne Fachgesellschaften haben bereits Untersuchungsbögen (DEGUM) erstellt [12]. Eine solche Sonografie ist aber nur in einer Infektionssprechstunde oder in den Corona-Bereitschaftspraxen sinnvoll und unter ausreichenden hygienischen Bedingungen durchführbar.

\section{Merke}

Die Pulsoxymetrie mit Dokumentation der Atemfrequenz ist eine weitere sinnvolle und umsetzbare diagnostische Methode im Bereich der Primärversorgung.

In der Notaufnahme erfolgt zusätzlich in der Regel eine radiologische Bildgebung bei Zeichen einer Infektion der unteren Atemwege. Zur Einschätzung der Schwere der Erkrankung hat sich hier vor allem das HR-CT der Lunge bewährt.

\section{Risikofaktoren}

COVID-19 kann sehr variabel verlaufen, und eine Verschlechterung auch bei anfänglich nur geringer Symptomatik ist möglich. Eine entscheidende Frage bei Diagnosestellung ist, ob eine stationäre Einweisung erfolgen sollte. Eine Liste von publizierten Risikofaktoren zeigt die Infobox $3[1,11]$. 


\section{INFOBOX 3}

Risikofaktoren:

- höheres Alter (vor allem > 65 Jahre)

- Übergewicht (BMI > $\left.30 \mathrm{~kg} / \mathrm{m}^{2}\right)$

- männliches Geschlecht

- chronische Lungenerkrankung

- Diabetes mellitus

- kardiovaskuläre Vorerkrankungen

- Immunsuppression

- schwere Niereninsuffizienz

Für die Einschätzung des Erkrankungsverlaufs haben sich die bisher verwendeten Scoring-Systeme (z. B. CRB-65) leider als wenig hilfreich erwiesen. Es wird unterschieden zwischen Risikofaktoren, die die Notwendigkeit eines Krankenhausaufenthalts wahrscheinlich machen, und solchen, die einen kritischen Krankheitsverlauf oder Tod vorhersagen. Natürlich bestehen hier Überlappungen. Die vermutlich mit Abstand wichtigsten Risikofaktoren für die Notwendigkeit einer stationären Behandlung sind höheres Alter, Übergewicht und männliches Geschlecht [13]. Prospektiv bestätigte Risiko-Scores existieren derzeit jedoch nicht. Für die Vorhersage eines kritischen Verlaufs eignen sich Parameter wie

- $\mathrm{SpO}_{2}$,

- Alter,

- Troponin-Erhöhung und

- erhöhtes PCT und CRP [13]

In unserer klinischen Erfahrung stellen vor allem erhöhte Entzündungszeichen (CRP > $97 \mathrm{mg} / \mathrm{l}$ und/oder IL-6> $80 \mathrm{pg} / \mathrm{ml}$ ) in der Notaufnahme ein entscheidendes Kriterium für einen komplizierten Erkrankungsverlauf dar [14].

\section{Merke \\ Speziell Patienten mit verminderter SpO2 bei Raumluft (<88\%) sowie erhöhten Entzündungszeichen sollten engmaschig überwacht werden.}

\section{Weitere Betreuung}

Eine enge Absprache zwischen Praxen, Notaufnahme und Normal- sowie Intensivstationen ist bei hohem Aufkommen von COVID-19-Patienten wichtig. Versorgungsengpässe müssen vermieden werden, um Patienten rasch und effizient je nach Risikoprofil zu verteilen. Patienten, die

- sich an die Hygieneregeln halten,

- nicht in einer Gemeinschaftsunterkunft leben und

- keine Hochrisikomerkmale aufweisen,

sollten möglichst ambulant zu Hause betreut werden, wenn sie klinisch stabil sind. Dazu ist eine enge Abstimmung zwischen ambulant tätigen Kollegen, Patienten, Gesundheitsamt und (bei Verschlechterung) der aufneh- menden Notaufnahme notwendig. Enge regionale Kontakte und kollegiale Zusammenarbeit sind hier für eine effiziente Versorgung entscheidend.

Einheitliche Empfehlungen bezüglich eines ambulanten Monitorings bestehen derzeit nicht. Für eine Krankenhauseinweisung sprechen unter anderem

- eine deutliche klinische Gesamtverschlechterung,

- Dyspnoe und

- eine Verminderung der Sauerstoffsättigung $\left(\mathrm{SpO}_{2}\right)$.

\section{Initiale Basis-Therapie bei COVID-19}

Auf die Darstellung spezifisch antiviral wirksamer Therapeutika sowie primär antiinflammatorischer Therapiekonzepte soll hier bewusst verzichtet werden. Diese Therapieansätze kommen in der Regel im Rahmen eines stationären Aufenthalts und vorwiegend im Rahmen klinischer Studien zum Einsatz. Allerdings wurden in den vergangenen Wochen immer wieder Ratschläge und Gerüchte über den Einfluss verschiedener Medikamente auf den Verlauf von COVID-19 berichtet, die zum Teil für große Verwirrung sorgten. Es soll deshalb hier vor allem auf die initiale und ambulante Basisbehandlung von COVID19 eingegangen werden.

\section{Merke}

Es existiert derzeit keine spezifische Basistherapie für Patienten mit COVID-19. Die Therapie richtet sich nach der Schwere der Erkrankung und besteht in der Regel aus supportiven Maßnahmen.

\section{Antibiotische Therapie}

Da bei einem Großteil der Patienten die Infektion nahezu asymptomatisch oder oligosymptomatisch verläuft, sind häufig überhaupt keine therapeutischen Maßnahmen notwendig. Eine routinemäßige antibiotische Therapie zur Prophylaxe einer sekundären bakteriellen Infektion sollte nicht erfolgen. Bei Verdacht auf eine bakterielle Superinfektion sollte eine antibiotische Therapie entsprechend den allgemeinen Empfehlungen zur Therapie der ambulant erworbenen Pneumonie begonnen werden.

\section{Antipyretische Therapie}

Sofern erforderlich können zur antipyretischen Therapie Metamizol oder NSAR verabreicht werden. Im Zusammenhang mit COVID-19 wurde immer wieder auf erhöhte Risiken durch die Einnahme von Ibuprofen hingewiesen. So hatte die Weltgesundheitsorganisation WHO vorübergehend von der Einnahme von Ibuprofen abgeraten. Diese Empfehlung wurde allerdings nach kurzer Zeit wieder zurückgezogen: Die Warnungen basierten auf der Veröffentlichung einer theoretischen Überlegung, dass bestimmte Medikamente die Ansteckung mit SARS-CoV-2 erleichtern könnten [15]. Aktuell gibt es keine Hinweise, dass die Einnahme von Ibuprofen über die bekannten Nebenwirkungen hinaus den Verlauf einer Infektion beeinflussen würde. 


\section{Vorbestehende medikamentöse Therapie}

Vor einem Absetzen einer vorbestehenden Therapie mit NSAR oder einer immunsuppressiven Therapie aufgrund beispielsweise einer rheumatologischen Erkrankung ohne triftige Gründe muss gewarnt werden.

Merke

Eine Reduktion oder das Absetzen einer vorbestehenden immunsuppressiven Therapie bei rheumatischen Erkrankungen ist mit einem erhöhten Risiko von Rezidiven verbunden.

Derartige Rezidive oder auch akute Schübe der Grunderkrankung erhöhen nicht nur das Risiko einer viralen Infektion, sondern führen durch die notwendige Intensivierung der immunsuppressiven Therapie letztlich zu höheren Medikamentendosierungen [16]. Ähnliches gilt für die antihypertensive Therapie mit ACE-Hemmern oder Sartanen. Fachgesellschaften wie die European Society of Cardiology (ESC) und die Deutsche Gesellschaft für Kardiologie (DGK) raten dringend davon ab, bestehende Therapien aufgrund theoretischer Überlegungen abzubrechen.

\section{Antikoagulanzien}

COVID-19 selbst bewirkt Koagulopathien mit thromboembolischen Komplikationen vor allem bei schweren und sehr schweren Verläufen. Patienten, die aufgrund COVID-19 stationär behandelt werden, sollten daher bei fehlenden Kontraindikationen mit einer medikamentösen Thromboseprophylaxe behandelt werden [17]. Bei einer leicht verlaufenden Infektion scheint das Risiko für thromboembolische Komplikationen nicht oder allenfalls gering erhöht zu sein. Eine Indikation für eine medikamentöse Thromboseprophylaxe bei ambulant behandelten Patienten besteht daher nicht.

\section{Verlauf und Genesung}

Eine regelhafte Testung aller entlassenen Patienten im ambulanten Bereich erscheint nicht sinnvoll. Werden dennoch bei diesen Patienten Testungen auf SARS-CoV-2 durchgeführt, dann ist im Falle eines positiven Befundes nicht zwingend davon auszugehen, dass damit auch das Vorhandensein größerer Mengen von vermehrungsfähigen Viren und eine Infektiosität für Dritte einhergeht. Das Gesundheitsamt muss hier den jeweiligen Einzelfall bewerten.

\section{Merke}

Ambulant behandelte Patienten, die mindestens 14 Tage in Quarantäne verbracht haben und mindestens 48 Stunden fieberfrei waren, können ohne weitere Testung und Diagnostik in ihr soziales Umfeld zurückkehren.

In Bezug auf die Rückkehr an den Arbeitsplatz ist auf entsprechende betriebsspezifische Regelungen zu achten (siehe RKI-Empfehlungen). Länger andauernde Verläufe der Erkrankung mit ausgeprägter Müdigkeit und reduzierter Leistungsfähigkeit führen häufig zu psychischen Belastungen und bedeuten für die ambulante Versorgung eine Herausforderung.

\section{KERNAUSSAGEN}

- Die Versorgung von Corona-Infizierten kann an der Schnittstelle ambulant/stationär verbessert werden. Es kann nach der Krise kein „weiter so“ geben!

- Ob ein Patient ambulant oder stationär behandelt werden muss, ist und bleibt eine Herausforderung und individuelle Entscheidung.

- Alter, männliches Geschlecht und Übergewicht gehören zu den wichtigsten Risikofaktoren einer schweren Corona-Erkrankung.

- Schlechte Sauerstoffsättigung (<88\%) und erhöhte Entzündungszeichen (CRP > 97 mg/l und/ oder IL-6> 80 pg/ml) sprechen für einen kritischen Verlauf und sollten bei symptomatischen Patienten bestimmt werden.

- Nur im regelmäßigen Dialog von Krankenhaus und Praxis können sinnvolle Entscheidungen gefällt werden, um langsam vom Einzelfall zu einer grundlegenden Versorgungsstruktur zu gelangen.

\section{Interessenkonflikt}

Die Autorinnen/Autoren geben an, dass kein Interessenkonflikt besteht.

\section{Autorinnen/Autoren}

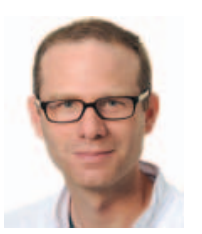

PD Dr. med. Tobias Herold

ist Facharzt für Innere Medizin und Hämatologie und Onkologie, Intensiv- und Palliativmediziner und Oberarzt der Zentralen Notaufnahme Großhadern (ZNA) am Klinikum der Universität München am Campus Großhadern.

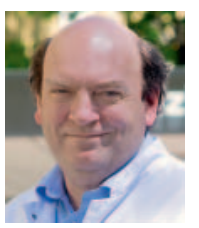

\section{Prof. Dr. med. Markus Wörnle}

ist Facharzt für Innere Medizin, Nephrologie, Angiologie und Intensivmedizin und Leiter der Notaufnahme am Campus Innenstadt, LMU Klinikum München.

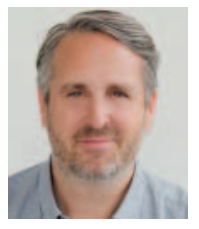

\section{Prof. Dr. med. habil. Jörg Schelling}

ist seit 2008 niedergelassen als Facharzt für Allgemeinmedizin in eigener Praxis. Ehemals Direktor des Instituts für Allgemeinmedizin der LMU München. Schwerpunkte: Impf- und Reisemedizin, Sonografie und Infektiologie in der Hausarztpraxis. 
Korrespondenzadresse

PD Dr. med. Tobias Herold

Klinikum der Universität München, Campus Großhadern

Zentrale Notaufnahme Großhadern (ZNA)

Marchioninistraße 15

81377 München

tobias.herold@med.uni-muenchen.de

Literatur

[1] Gandhi RT, Lynch JB, Del Rio C. Mild or Moderate Covid-19. N Engl ] Med 2020. doi:10.1056/NEJMcp2009249

[2] Kassenärztliche Vereinigung Bayerns. Infektionsschutz COVID19: Informationen für Ärzte. Im Internet (Stand: 10.06.2020): www.kvb.de/praxis/qualitaet/hygiene-und-infektionspraeventi on/infektionsschutz/coronavirus/

[3] Deutsche Gesellschaft für Allgemeinmedizin und Familienmedizin (DEGAM). Im Internet (Stand:10.06.2020): www.degam. de/

[4] Kassenärztliche Vereinigung Bayerns. Patienteninformation Coronavirus - vom Test bis zur Genesung. Im Internet (Stand 10.06.2020): www.kvb.de/fileadmin/kvb/dokumente/Praxis/In fomaterial/GesundheitPraevention/KVB-Patienteninfoblatt-Co ronavirus-Tests.pdf

[5] Staatsministerium für Gesundheit und Pflege, Staatsministerium des Innern, für Sport und Integration und Kassenärztliche Vereinigung Bayerns. Gemeinsames Konzept zur Einrichtung und Betrieb von Schwerpunktpraxen (Stand: 11.04.2020). Im Internet (Stand 10.06.2020): www.stmgp.bayern.de/wp-con tent/uploads/2020/04/20200411_konzept_schwerpunktpra xen.pdf

[6] Lauer SA, Grantz KH, Bi Q et al. The Incubation Period of Coronavirus Disease 2019 (COVID-19) From Publicly Reported Confirmed Cases: Estimation and Application. Ann Intern Med 2020. doi:10.7326/M20-0504

[7] Pan L, Mu M, Yang P et al. Clinical Characteristics of COVID-19 Patients With Digestive Symptoms in Hubei, China: A Descriptive, Cross-Sectional, Multicenter Study. Am J Gastroenterol 2020; 115: 766-773. doi:10.14309/ajg.0000000000000620

[8] Wang D, Hu B, Hu C et al. Clinical Characteristics of 138 Hospitalized Patients With 2019 Novel Coronavirus-Infected Pneumonia in Wuhan, China. JAMA 2020. doi:10.1001/jama.2020.1585

[9] Lechien JR, Chiesa-Estomba CM, De Siati DR et al. Olfactory and gustatory dysfunctions as a clinical presentation of mild-tomoderate forms of the coronavirus disease (COVID-19): a multicenter European study. Eur Arch Otorhinolaryngol 2020. doi:10.1007/s00405-020-05965-1
[10] Wang W, Xu Y, Gao R et al. Detection of SARS-CoV-2 in Different Types of Clinical Specimens. JAMA 2020. doi:10.1001/jama.2020.3786

[11] Zhou F, Yu T, Du R et al. Clinical course and risk factors for mortality of adult inpatients with COVID-19 in Wuhan, China: a retrospective cohort study. Lancet 2020; 395: 1054-1062. doi:10.1016/50140-6736(20)30566-3

[12] Deutsche Gesellschaft für Ultraschall in der Medizin (DEGUM). Vorschlag eines standardisierten Lungen-Ultraschallprotokolls in den Zeiten von COVID-19. Im Internet (Stand 10.06.2020): www.degum.de/en/nc/aktuelles/degum-ultraschall/im-de tail/news/anleitung-zum-lungenultraschall-bei-verdacht-aufcovid-19.html

[13] Petrilli CM, Jones SA, Yang J et al. Factors associated with hospitalization and critical illness among 4103 patients with COVID-19 disease in New York City. medRxiv 2020. doi:10.1101/ 2020.04.08.20057794

[14] Herold T, Jurinovic V, Arnreich C et al. Elevated levels of interleukin- 6 and CRP predict the need for mechanical ventilation in COVID-19. J Allergy Clin Immunol 2020. doi:10.1016/j.jaci.2020.05.008

[15] Fang L, Karakiulakis G, Roth M. Are patients with hypertension and diabetes mellitus at increased risk for COVID-19 infection? Lancet Respir Med 2020; 8: e21. doi:10.1016/S22132600(20)30116-8

[16] Schulze-Koops H, Holle J, Moosig F et al. Current guidance of the German Society of Rheumatology for the care of patients with rheumatic diseases during the SARS-CoV-2/Covid 19 pandemic. Z Rheumatol 2020. doi:10.1007/s00393-02000799-y

[17] Paranjpe I, Fuster V, Lala A et al. Association of Treatment Dose Anticoagulation with In-Hospital Survival Among Hospitalized Patients with COVID-19. J Am Coll Cardiol 2020. doi:10.1016/j.jacc.2020.05.001

Bibliografie

DOI https://doi.org/10.1055/a-1164-4130 Dtsch Med Wochenschr 2020; 145: 1080-1085 (c) Georg Thieme Verlag KG, Stuttgart · New York ISSN 0012-0472 